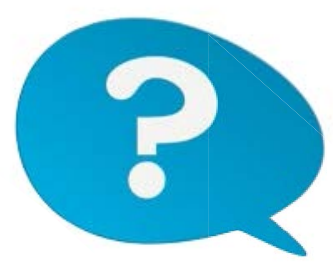

\title{
Wie kann das Ramsay Hunt Syndrom therapiert werden?
}

Frage: Verbessert die frühzeitige antivirale Behandlung die Prognose bei Zoster oticus? Gibt es Erkenntnisse bezüglich der Verbesserung von Fazialisparese, Hörminderung oder Schwindel? Welche antiviralen Therapeutika kommen hierfür infrage und welche Dosierung, Therapiedauer und welchen Darreichungsweg (p.o./i.v.) empfehlen Sie?

Antwort: Als Ramsay Hunt Syndrom (RHS) wird ein Zoster oticus in Verbindung mit akuter Fazialisparese sowie einer Assoziation mit vestibulärcochlearer Dysfunktion bezeichnet. Ursache ist eine Reaktivierung des Varizella-zosterVirus (VZV) im Ganglion geniculatum. Ein RHS ist bei Kindern unter sechs Jahren selten, bei älteren Kindern und Erwachsenen ist die Inzidenz ähnlich [1]. Bei Kindern treten die Zosterbläschen am Ohr in ca. $15 \%$ vor, in ca. $34 \%$ simultan und in ca. $50 \%$ nach Beginn der Fazialisparese auf [1]. Unterschiedlich häufig ist der N. VIII betroffen (Tab. 1)
Die Therapie orientiert sich national und international letztlich an den Empfehlungen der Leitlinie der Deutschen Gesellschaft für Neurologie bei idiopathischer Fazialisparese (Bell's palsy) [2].

Standardtherapie des RHS ist eine Therapie mit hochdosierten Kortikosteroiden und Aciclovir, möglichst begonnen innerhalb der ersten drei Krankheitstage:

Aciclovir (3 x tgl. i. v. $5-10 \mathrm{mg} / \mathrm{kg} \mathrm{KG}$ oder $5 \mathrm{x}$ tgl. p. o. $800 \mathrm{mg}$ ) wird empfohlen für 7-10 Tage, zusammen mit potenten antiinflammatorischen Steroiden (Prednisolon initial $1 \mathrm{mg} / \mathrm{kg} \mathrm{KG/d} \mathrm{p.} \mathrm{o.} \mathrm{oder} \mathrm{Methyl-}$ prednisolon $20-30 \mathrm{mg} / \mathrm{kg}$ KG/d während der ersten drei bis fünf Tage), danach werden die Substanzen über eine Woche oder länger ausgeschlichen. Die antientzündliche, antiödematöse Steroidtherapie reduziert wahrscheinlich Schmerz und Vertigo [3]. Valaciclovir und Famciclovir sind für VZV-Infektionen erst ab einem Alter von 18 Jahren zugelassen.

In einer kürzlichen Metaanalyse an erwachsenen Patienten mit RHS lag die the-

Tab. 1: Beteiligung des N. VIII bei Zoster oticus [1]

\begin{tabular}{|l|l|l|l|}
\hline & Hörstörung & Tinnitus & Vertigo \\
\hline Kinder & $11 / 45(24,4 \%)$ & $2 / 18(11,1 \%)$ & $4 / 23(17,4 \%)$ \\
\hline Erwachsene & $125 / 237(52,7 \%)$ & $40 / 162(24,7 \%)$ & $50 / 157(31,8 \%)$ \\
\hline
\end{tabular}

\section{Haben Sie auch eine fachliche Frage?}

Das "Consilium HNO" ist ein Service des Unternehmens Infectopharm. Als HNO-Arzt haben Sie die Möglichkeit, selbst Fragen zum gesamten Fachgebiet zu stellen. Schreiben Sie dazu an Dr. Kristin Brendel-Walter, Von-Humboldt-Straße 1, 64646 Heppenheim, oder schicken Sie eine E-Mail an consilium@infectopharm.com. Ihre Frage wird anonym an ein Mitglied des Expertenpools weitergeleitet, Sie erhalten eine schriftliche Antwort.

Wenn Sie sehen wollen, was die Kolleginnen und Kollegen so fragen, können Sie im Archiv des "Consilium HNO" unter www.infectopharm.com/consilium stöbern. Außerdem werden redaktionell ausgewählte, allgemein interessante Anfragen zusätzlich in der Zeitschrift „HNO-Nachrichten“ publiziert. rapeutische Erfolgsrate (House-Brackmann (HB) Grad II, komplette Remission) ohne Therapie bei 0,38 , unter Aciclovir bei 0,77 , unter Steroiden bei 0,65 und unter einer Kombinationstherapie (Aciclovir + Steroid) bei 0,82 [4] bzw. 0,84 [5]. Bezüglich Kindern und Jugendlichen gibt es keine kontrollierten Studien. Die Therapieansätze orientieren sich an den Erwachsenendaten.

Die frühzeitige (altersentsprechende) VZV-Impfung kann bei einem Großteil der Kinder eine VZV-Primärinfektion verhindern. Ob auch das VZ-Impfvirus bei möglicher Reaktivierung später ein Ramsay Hunt Syndrom verursachen kann, ist derzeit nicht bekannt. In einem kürzlich publizierten Fall aus Japan hatte die frühzeitige VZV-Impfung ein späteres RHS (Wildvirusinfektion) nicht verhindert [6].

\section{Prof. Dr. med. Volker Schuster}

Universitätsklinikum Leipzig

Liebigstr. 20a, 04103 Leipzig

\section{Literatur}

1. Hato N, Kisaki H, Honda N, Gyo K, Murakami $S$, Yanagihara N. Ramsay Hunt syndrome in children. Ann Neurol 2000, 48 (2), 254-56.

2. Heckmann JG, Lang C, Glocker FX, Urban P, Bischoff C et al. Leitlinie der Deutschen Gesellschaft für Neurologie - Therapie der idiopathischen Fazialisparese (Bell's palsy). AMWF 2011, 030/013.

3. Kansu L, Yilmaz I. Herpes zoster oticus (Ramsay Hunt syndrome) in children: case report and literature review. Int J Pediatr Otorhinolaryngol 2012, 76 (6), 772-76.

4. Uri N, Greenberg E, Kitzes-Cohen R, Doweck I. Aciclovir in the treatment of Ramsay Hunt syndrome. Otolaryngol Head Neck Surg 2003, 129 (4), 379-81.

5. de Ru JA, van Benthem PP. Combination therapy is preferable for patients with Ramsay Hunt syndrome. Otol Neurotol 2011, 32 (5), 852-55.

6. Nakamura Y, Matsumoto H. Case of atypical Ramsay-Hunt syndrome who presented with severe vertigo and vomiting. No To Hattatsu 2012, 44 (1), 66-68. 